\title{
Effectiveness Of Nursing Competency Of Service Quality In Installation Of Hospital Buleleng Hospital
}

Made Susiana
Magister of Health Study Program of
Institut Ilmu Kesehatan STRADA
Indonesia
Email:
publikasistrada@gmail.com

Received : October 12, 2019

Accepted : February 13, 2020

Published : May 12, 2020

\begin{abstract}
Buleleng Regional General Hospital as a health service provider is concerned with the quality of its services. The quality of nurse services is the biggest part of the overall quality of hospital services because of the amount of contact with patients. The quality of service itself is influenced by the satisfaction and expectations and needs of patients being met. To meet the excellent quality of service, nurses need to have superior competence, namely basic knowledge, hard skills, soft skills, work ethics and legal aspects. The purpose of this study is to provide recommendations based on an analysis of the influence of nurse characteristics and competence on service quality. This research is a descriptive observational study, conducted from November. The study population was nurses working in the Inpatient Room, and the number of samples obtained was 80 nurses. The variables analyzed were individual characteristics, nurse competence, hard skills, soft skills, work ethics, and legal aspects. The results of this study indicate that age, length of work and position of head of the family at work have an influence on nurse competence. Length of work, and soft skills also have a positive influence on service quality. Conclusion: there is a significant effect between length of work, and nurses' soft skills on service quality. Variables that need to be improved and improved are registration letters, work licenses, training, and training to improve soft skills and work ethics
\end{abstract}

Keywords: Competence, Service Quality, Hospitalization 


\section{INTRODUCTION}

The hospital as an institution engaged in the field of health services continues to change. The basic nature of the hospital is the fulfillment of the needs and expectations of patients to solve their health problems at the hospital. Quality service will be achieved if all human resources (hereinafter referred to as HR) have professional competence, understand products in depth, be friendly and friendly, responsive or sensitive to patients, master the work, communicate effectively and be able to respond to patient complaints and look attractive. Patient satisfaction starts from the time of arrival until the patient leaves the hospital. To create patient loyalty to the hospital, patient satisfaction must reach a special stage because the value of satisfaction in the ordinary category is very easy to fall or turn bad, which can easily occur if the quality of service received does not match the needs and expectations of the patient. The presence of ordinary and even poor nurse services is evidence that nurses' competencies need to get attention, especially on the soft competency element because of the decrease in the level of satisfaction with the services provided by nurses on caring elements in providing services to patients. Nurses as service providers must be updated and upgraded to be able to meet the needs and expectations of patients in providing services. Nurse competence is an important aspect in the quality of service that contains the consequences of authority in the task as well as making decisions according to their roles that are relevant to expertise, knowledge and skills. Nurse competence is needed to support the hospital strategy in maintaining and improving the quality of service for patient satisfaction. The purpose of this study is to develop recommendations for improving service quality based on an analysis of the influence of nurse competencies with service quality in Inpatient Installation.

\section{METHODS}

This research belongs to the descriptive observational category. The researcher will analyze the effect of the nurse competency variable on the quality of nurse services in the inpatient room of Buleleng Regional Hospital. This study uses a cross sectional design. In this study, researchers conducted a study on the characteristics of individual nurses, nurse competencies in inpatient installations, and conducted an analysis of the quality of nurse services in inpatient installations, as well as testing the effect on both variables. This research was conducted in 8 (eight) Buleleng IRNA treatment rooms, namely: Emerald treatment room (surgical medical), Ruby treatment room (surgical medical), Sapphire treatment room (children), Pyrus treatment room (obstetrics and gynecology), Diamond treatment room (surgical medical), Pearl treatment room (surgical medical), Mirah treatment room (surgical medical), Emerald treatment room (surgical medical).

This research was conducted from March to December in 8 (eight) inpatient treatment rooms of Buleleng Regional Hospital. The stages of this research began with the preparation of proposals, preproposal seminars, preparation of research instruments, trial of research instruments, preparation of proposals, data collection, data processing, data analysis, preparation of research reports and completion of final reports. The population in this study were individual nurses in 8 (eight) treatment rooms. The total number of nurses is 120 people. The sample size used in this study used the Correlative Formula with the transformation of the $\mathrm{Z}$ value. By using this formula, a sample size of 79.86 was rounded up to 80 nurse samples. Sampling in this study uses a simple random sampling method that is random sampling. Validity measurement is done by comparing the results of the calculation of the value of corrected items - total correlation with the values in table $r$. Test for normality to see the data that has gathered normal distribution or not. Performed on soft competency variables, work ethics and the quality of nurse services to be tested.

\section{RESULTS}

A. Characteristics of individual nurses

\section{Age's Nurse's}

Table 5.1 Distribution Age's Nurse's

\begin{tabular}{|l|l|r|r|}
\hline No & \multicolumn{1}{|c|}{ Categories Age's } & Amount & Percentage \\
\hline 1. & $20-25$ years & 17 & $21,3 \%$ \\
\hline 2. & $26-30$ years & 49 & $61,3 \%$ \\
\hline 3. & $>30$ years & 14 & $17,5 \%$ \\
\hline \multicolumn{2}{|l|}{ Total }
\end{tabular}




\section{Nurse's Service Period}

Table 5.2 Distribution Years of Service Nurse's

\begin{tabular}{|l|l|r|r|}
\hline No & \multicolumn{1}{|c|}{ Categories Years of Service } & Amount & Percentage \\
\hline 1. & $<5$ years & 51 & $63,7 \%$ \\
\hline 2. & $5-10$ years & 24 & $30,0 \%$ \\
\hline 3. & $>10$ years & 5 & $6,3 \%$ \\
\hline \multicolumn{2}{|l|}{ Total }
\end{tabular}

\section{Extra IncomeNurse's}

Table 5.3 Distribution Extra IncomeNurse's

\begin{tabular}{|l|l|r|r|}
\hline No & Categories Extra Income & Amount & \multicolumn{1}{|c|}{ Percentage } \\
\hline 1. & $<\operatorname{Rp~3.000.000~}$ & 30 & $37,5 \%$ \\
\hline 2. & Rp 3.000.000 - Rp 5.999.999 & 45 & $56,3 \%$ \\
\hline 3. & Rp 6.000.000 - Rp 8.999.999 & 3 & $3,8 \%$ \\
\hline 4. & $\geq \operatorname{Rp~9.000.000~}$ & 2 & $2,5 \%$ \\
\hline \multicolumn{2}{|l|}{ Total }
\end{tabular}

4. Position Head of Family Nurse's

Table 5.4 Distribution Position Head of Family Nurse's

\begin{tabular}{|l|l|r|r|}
\hline No & \multicolumn{1}{|c|}{ Categories Position } & Amount & \multicolumn{1}{|c|}{ Percentage } \\
\hline 1. & No Having Position & 26 & $32,5 \%$ \\
\hline 2. & Staff Level & 49 & $61,3 \%$ \\
\hline 3. & Unit Head Level & 5 & $6,3 \%$ \\
\hline \multicolumn{2}{|l|}{ Total } & 80 & $100,0 \%$ \\
\hline
\end{tabular}

\section{B. Factor of Basic Knowledge Nurse's}

1. Education of Nurse's

Table 5.5 Distribution Education of Nurse's

\begin{tabular}{|l|l|r|r|}
\hline No & Categories Education of Nurse's & Amount & Percentage \\
\hline 1. & Diploma & 64 & $80,0 \%$ \\
\hline 2. & Bachelor & 16 & $20,0 \%$ \\
\hline \multicolumn{2}{|l|}{ Total } & 80 & $100,0 \%$ \\
\hline
\end{tabular}

2. Trining Nurse's

Table 5.6 Distribution Trining Nurse's

\begin{tabular}{|l|l|r|r|}
\hline No & \multicolumn{1}{|c|}{ Categories Trining } & Amount & \multicolumn{1}{c|}{ Percentage } \\
\hline 1. & $<10$ time's & 38 & $47,5 \%$ \\
\hline 2. & $10-20$ time's & 39 & $48,8 \%$ \\
\hline 3. & $>20$ time's & 3 & $3,8 \%$ \\
\hline \multicolumn{2}{|l|}{ Total }
\end{tabular}

C. Factor of Hard Skill Nurse's

Table 5.7 Result of Hard Skill Nurse's

\begin{tabular}{|l|l|r|r|}
\hline No & \multicolumn{1}{|c|}{ Categories Hard Skill } & Amount & \multicolumn{1}{|c|}{ Percentage } \\
\hline 1. & Meet Standards & 74 & $92,5 \%$ \\
\hline 2. & Not Meeting the Standards & 6 & $7,5 \%$ \\
\hline Total & 80 & $100,0 \%$ \\
\hline
\end{tabular}

D. Factor of Soft Skill Nurse's

1. Personal Effectiveness of Nurse's

Table 5.8 Personal Effectiveness Nurse's

\begin{tabular}{|c|l|r|r|}
\hline No & Categories Personal Effectiveness & Amount & Percentage \\
\hline 1 & Good & 22 & $27,5 \%$ \\
\hline 2 & Bad & 58 & $72,5 \%$ \\
\hline
\end{tabular}




\begin{tabular}{|l|r|r|}
\hline Total & 80 & $100,0 \%$ \\
\hline
\end{tabular}

2. Flexibility Nurse's

Table 5.9 Flexibility of Nurse's

\begin{tabular}{|c|l|r|r|}
\hline No & Categories Flexibility & Amount & Percentage \\
\hline 1 & Good & 21 & $26,3 \%$ \\
\hline 2 & Bad & 59 & $73,7 \%$ \\
\hline Total & 80 & $100,0 \%$ \\
\hline
\end{tabular}

3. Leadership of Nurse's

Table 5.10 Leadership of Nurse's

\begin{tabular}{|c|l|r|r|}
\hline No & Categories Leadership & Amount & \multicolumn{1}{|c|}{ Percentage } \\
\hline 1 & Good & 7 & $8,8 \%$ \\
\hline 2 & Bad & 73 & $91,3 \%$ \\
\hline Total & 80 & $100,0 \%$ \\
\hline
\end{tabular}

4. Persuasion Nurse's

Table 5.11 Persuasion Nurse's

\begin{tabular}{|c|l|r|r|}
\hline No & Categories Persuasion & Amount & \multicolumn{1}{|c|}{ Percentage } \\
\hline 1 & Good & 15 & $18,8 \%$ \\
\hline 2 & Bad & 65 & $81,2 \%$ \\
\hline Total & 80 & $100,0 \%$ \\
\hline
\end{tabular}

5. Goal Orientation Nurse's

Table 5.12 Goal Orientation Nurse's

\begin{tabular}{|c|l|r|r|}
\hline No & Categories Goal Orientation & Amount & Percentage \\
\hline 1 & Good & 18 & $22,5 \%$ \\
\hline 2 & Bad & 62 & $77,5 \%$ \\
\hline Total & & 80 & $100,0 \%$ \\
\hline
\end{tabular}

6. Decision Making of Nurse's

Table 5.13 Decision Making of Nurse's

\begin{tabular}{|c|l|r|r|}
\hline No & Categories Decision Making & Amount & Percentage \\
\hline 1 & Good & 14 & $17,5 \%$ \\
\hline 2 & Bad & 66 & $82,5 \%$ \\
\hline Total & 80 & $100,0 \%$ \\
\hline
\end{tabular}


7. Negotiation Nurse's

Table 5.14 Negotiation Nurse's

\begin{tabular}{|c|l|r|r|}
\hline No & \multicolumn{1}{|c|}{ Categories Negotiation } & Amount & Percentage \\
\hline 1 & Good & 17 & $21,3 \%$ \\
\hline 2 & Bad & 63 & $78,7 \%$ \\
\hline Total & 80 & $100,0 \%$ \\
\hline
\end{tabular}

8. Analytical Problem Solving Nurse's

Table 5.15 Analytical Problem Solving Nurse's

\begin{tabular}{|c|l|r|r|}
\hline No & Categories Analytical Problem Solving & Amount & \multicolumn{1}{|c|}{ Percentage } \\
\hline 1 & Good & 15 & $18,8 \%$ \\
\hline 2 & Bad & 65 & $81,2 \%$ \\
\hline Total & 80 & $100,0 \%$ \\
\hline
\end{tabular}

9. Teamwork Nurse's

Table 5.16 Teamwork Nurse's

\begin{tabular}{|c|l|r|r|}
\hline No & Categories Teamwork & Amount & Percentage \\
\hline 1 & Good & 24 & $30,0 \%$ \\
\hline 2 & Bad & 56 & $70,0 \%$ \\
\hline Total & 80 & $100,0 \%$ \\
\hline
\end{tabular}

10. Diplomacy Nurse's

Table 5.17 Diplomacy Nurse's

\begin{tabular}{|c|l|r|r|}
\hline No & Categories Diplomacy & Amount & Percentage \\
\hline 1 & Good & 17 & $21,3 \%$ \\
\hline 2 & Bad & 63 & $78,7 \%$ \\
\hline Total & 80 & $100,0 \%$ \\
\hline
\end{tabular}

11. Empathy Nurse's

Table 5.18 Empathy Nurse's

\begin{tabular}{|c|l|r|r|}
\hline No & Categories Empathy & Amount & Percentage \\
\hline 1 & Good & 16 & $20,0 \%$ \\
\hline 2 & Bad & 64 & $80,0 \%$ \\
\hline Total & 80 & $100,0 \%$ \\
\hline
\end{tabular}

12. Interpersonal Skill Nurse's

Table 5.19 Interpersonal Skill Nurse's Years

\begin{tabular}{|c|l|r|r|}
\hline No & Categories Interpersonal Skill & Amount & Percentage \\
\hline 1 & Good & 24 & $30,0 \%$ \\
\hline 2 & Bad & 56 & $70,0 \%$ \\
\hline Total & 80 & $100,0 \%$ \\
\hline
\end{tabular}

13. Self Management (Time And Priorities) Nurse's

Table 5.20 Self Management (Time And Priorities) Nurse's Years

\begin{tabular}{|c|l|r|r|}
\hline No & Categories Self Management (Time and Priorities) & Amount & Percentage \\
\hline 1 & Good & 12 & $15,0 \%$ \\
\hline 2 & Bad & 68 & $85,0 \%$ \\
\hline Total & 80 & $100,0 \%$ \\
\hline
\end{tabular}


14. Soft Skill Nurse's

Table 5.21 Soft Skill Nurse's

\begin{tabular}{|c|l|r|r|}
\hline No & Categories Soft Skill & Amount & Percentage \\
\hline 1 & Good & 16 & $20,0 \%$ \\
\hline 2 & Bad & 64 & $80,0 \%$ \\
\hline Total & 80 & $100,0 \%$ \\
\hline
\end{tabular}

\section{E. Factor of Work Ethics Nurse's}

1. Self Reliance Nurse's

Table 5.22 Self Reliance Nurse's Years

\begin{tabular}{|c|l|r|r|}
\hline No & Categories Self Reliance & Amount & Percentage \\
\hline 1 & Good & 16 & $20,0 \%$ \\
\hline 2 & Bad & 64 & $80,0 \%$ \\
\hline Total & 80 & $100,0 \%$ \\
\hline
\end{tabular}

2. Morality Nurse's

Table 5.23 Morality Nurse's

\begin{tabular}{|c|l|r|r|}
\hline No & Categories Morality & Amount & Percentage \\
\hline 1 & Good & 46 & $57,5 \%$ \\
\hline 2 & Bad & 34 & $42,5 \%$ \\
\hline Total & 80 & $100,0 \%$ \\
\hline
\end{tabular}

3. Leisure Nurse's

Table 5.24 Leisure Nurse's

\begin{tabular}{|c|l|r|r|}
\hline No & Categories Leisure & Amount & Percentage \\
\hline 1 & Good & 1 & $1,2 \%$ \\
\hline 2 & Bad & 79 & $98,8 \%$ \\
\hline Total & 80 & $100,0 \%$ \\
\hline
\end{tabular}

4. Hard Work Nurse's

Table 5.25 Hard Work Nurse's

\begin{tabular}{|c|l|r|r|}
\hline No & Categories Hard Work & Amount & Percentage \\
\hline 1 & Good & 38 & $47,5 \%$ \\
\hline 2 & Bad & 42 & $52,5 \%$ \\
\hline Total & 80 & $100,0 \%$ \\
\hline
\end{tabular}

5. Centrality of Work Nurse's

Table 5.26 Centrality Of Work Nurse's

\begin{tabular}{|c|l|r|r|}
\hline No & Categories Centrality of Work & Amount & Percentage \\
\hline 1 & Good & 12 & $15 \%$ \\
\hline 2 & Bad & 68 & $85 \%$ \\
\hline Total & 80 & $100 \%$ \\
\hline
\end{tabular}

6. Wasted Time Nurse's

Table 5.27 Wasted Time Nurse's

\begin{tabular}{|c|l|r|r|}
\hline No & \multicolumn{1}{|c|}{ Categories Wasted Time } & Amount & \multicolumn{1}{|c|}{ Percentage } \\
\hline 1 & Good & 9 & $11,3 \%$ \\
\hline 2 & Bad & 71 & $88,7 \%$ \\
\hline Total & 80 & $100,0 \%$ \\
\hline
\end{tabular}


7. Delay of Gratification Nurse's

Table 5.28 Delay of Gratification Nurse's

\begin{tabular}{|c|l|r|r|}
\hline No & Categories Delay of Gratification & Amount & Percentage \\
\hline 1 & Good & 17 & $21,3 \%$ \\
\hline 2 & Bad & 63 & $78,7 \%$ \\
\hline Total & 80 & $100,0 \%$ \\
\hline
\end{tabular}

8. Work Ethics Nurse's

Table 5.29 Work Ethics Nurse's

\begin{tabular}{|c|l|r|r|}
\hline No & \multicolumn{1}{|c|}{ Categories Work Ethics } & Amount & Percentage \\
\hline 1 & Good & 7 & $8,8 \%$ \\
\hline 2 & Bad & 73 & $91,2 \%$ \\
\hline Total & 80 & $100,0 \%$ \\
\hline
\end{tabular}

F. Factor of Legal Aspect Nurse's

1. Nurses Competence Record

Table 5.30 Distribution Nurses Competence Record

\begin{tabular}{|c|l|r|r|}
\hline No & Categories Competence Result & Amount & Percentage \\
\hline 1 & One Times exam & 79 & $98,8 \%$ \\
\hline 2 & Two Times exam & 1 & $1,2 \%$ \\
\hline Total & 80 & $100,0 \%$ \\
\hline
\end{tabular}

2. Nurse's Registration Certificate(STR) Nurse's

Table 5.31 Distributuion Period of STR Nurse's

\begin{tabular}{|c|l|r|r|}
\hline No & \multicolumn{1}{|c|}{ Categories STR } & Amount & \multicolumn{1}{c|}{ Percentage } \\
\hline 1 & STR Still Valid & 72 & $90,0 \%$ \\
\hline 2 & STR Expiry date & 8 & $10,0 \%$ \\
\hline Total & & 80 & $100,0 \%$ \\
\hline
\end{tabular}

3. Nurse's Practic License(SIP) Nurse's

Table 5.32 Distributuion Period of SIP Nurse's

\begin{tabular}{|c|l|r|r|}
\hline No & \multicolumn{1}{|c|}{ Categories SIP } & Amount & Percentage \\
\hline 1 & SIP Still Valid & 72 & $90,0 \%$ \\
\hline 2 & SIP Expiry date & 8 & $10,0 \%$ \\
\hline Total & & 80 & 80 \\
\hline
\end{tabular}

G. Service Quality Nurse's

1. Knowledgeability Nurse's

Table 5.33 Knowledgeability Nurse's

\begin{tabular}{|c|l|r|r|}
\hline No & Categories Knowledgeability & Amount & Percentage \\
\hline 1 & Good & 30 & $37,5 \%$ \\
\hline 2 & Bad & 50 & $62,5 \%$ \\
\hline Total & 80 & $100,0 \%$ \\
\hline
\end{tabular}

2. Responsiveness Nurse's

Table 5.34 Responsiveness Nurse's

\begin{tabular}{|c|l|r|r|}
\hline No & Categories Responsiveness & Amount & Percentage \\
\hline 1 & Good & 36 & $45,0 \%$ \\
\hline 2 & Bad & 44 & $55,0 \%$ \\
\hline Total & 80 & $100,0 \%$ \\
\hline
\end{tabular}


3. Professionalism Nurse's

Table 5.35 Professionalism Nurse's Years

\begin{tabular}{|c|l|r|r|}
\hline No & Categories Professionalism & Amount & Percentage \\
\hline 1 & Good & 37 & $46,3 \%$ \\
\hline 2 & Bad & 43 & $53,7 \%$ \\
\hline Total & 80 & $100,0 \%$ \\
\hline
\end{tabular}

4. Accessibility Nurse's

Table 5.36 Accessibility Nurse's

\begin{tabular}{|c|l|r|r|}
\hline No & Categories Accessibility & Amount & Percentage \\
\hline 1 & Good & 39 & $48,8 \%$ \\
\hline 2 & Bad & 41 & $51,2 \%$ \\
\hline Total & 80 & $100,0 \%$ \\
\hline
\end{tabular}

5. Caring Nurse's

Table 5.37 Caring Nurse's

\begin{tabular}{|c|l|r|r|}
\hline No & Categories Caring & Amount & Percentage \\
\hline 1 & Good & 44 & $55,0 \%$ \\
\hline 2 & Bad & 36 & $45,0 \%$ \\
\hline Total & 80 & $100,0 \%$ \\
\hline
\end{tabular}

6. Dignity Nurse's

Table 5.38 Dignity Nurse's

\begin{tabular}{|c|l|r|r|}
\hline No & Categories Dignity & Amount & Percentage \\
\hline 1 & Good & 44 & $55,0 \%$ \\
\hline 2 & Bad & 36 & $45,0 \%$ \\
\hline Total & 80 & $100,0 \%$ \\
\hline
\end{tabular}

7. Empathy Nurse's

Table 5.39 Empathy Nurse's

\begin{tabular}{|c|l|r|r|}
\hline No & Empathy & Amount & Percentage \\
\hline 1 & Good & 34 & $42,5 \%$ \\
\hline 2 & Bad & 46 & $57,5 \%$ \\
\hline Total & & 80 & $100,0 \%$ \\
\hline
\end{tabular}

8. Convenience Nurse's

Table 5.40 Convenience Nurse's

\begin{tabular}{|c|l|r|r|}
\hline No & Categories Convenience & Amount & Percentage \\
\hline 1 & Good & 43 & $53,8 \%$ \\
\hline 2 & Bad & 37 & $46,3 \%$ \\
\hline Total & 80 & $100,0 \%$ \\
\hline
\end{tabular}

9. Information Nurse's

Table 5.41 Information Nurse's

\begin{tabular}{|c|l|r|r|}
\hline No & Categories Information & Amount & Percentage \\
\hline 1 & Good & 28 & $35,0 \%$ \\
\hline 2 & Bad & 52 & $65,0 \%$ \\
\hline Total & 80 & $100,0 \%$ \\
\hline
\end{tabular}


10. Communication Nurse's

Table 5.42 Communication Nurse's

\begin{tabular}{|c|l|r|r|}
\hline No & Categories Communication & Amount & Percentage \\
\hline 1 & Good & 38 & $47,5 \%$ \\
\hline 2 & Bad & 42 & $52,5 \%$ \\
\hline Total & 80 & $100,0 \%$ \\
\hline
\end{tabular}

11. Service Quality Nurse's

Table 5.43 Service Quality Nurse's

\begin{tabular}{|c|l|r|r|}
\hline No & Categories Service Quality & Amount & Percentage \\
\hline 1 & Service Quality Good & 42 & $52,5 \%$ \\
\hline 2 & Service Quality Bad & 38 & $47,5 \%$ \\
\hline Total & & 80 & $100,0 \%$ \\
\hline
\end{tabular}

1. Analysis of Individual Characteristics of Nurse's Competence

a. Analysis Age's Nurse's Education of Nurse's

Table 5.44 Cross Tabulation Age's With Education of Nurse's

\begin{tabular}{|c|c|c|c|c|c|c|c|}
\hline \multirow{3}{*}{ No } & \multirow{3}{*}{ Age's } & \multicolumn{4}{|c|}{ Education of } & \multirow{2}{*}{\multicolumn{2}{|c|}{ Total }} \\
\hline & & \multicolumn{2}{|c|}{ Bachelor } & \multicolumn{2}{|c|}{ Diploma } & & \\
\hline & & $\mathrm{n}$ & $\%$ & $\mathrm{n}$ & $\%$ & $\mathrm{n}$ & $\%$ \\
\hline 1. & $20-25$ years & 8 & 47,1 & 9 & 52,9 & 17 & 100,0 \\
\hline 2. & $26-30$ years & 7 & 14,3 & 42 & 85,7 & 49 & 100,0 \\
\hline 3. & $>30$ years & 1 & 7,1 & 13 & 92,9 & 14 & 100,0 \\
\hline \multicolumn{2}{|c|}{ Total } & 16 & 20,0 & 64 & 80,0 & 80 & 100,0 \\
\hline
\end{tabular}

b. Analysis Age's Nurse's Of Trining Nurse's

Table 5.45 Cross Tabulation Age's With Trining Nurse's

\begin{tabular}{|c|c|c|c|c|c|c|c|c|c|}
\hline \multirow{3}{*}{ No } & \multirow{3}{*}{ Age's } & \multicolumn{6}{|c|}{ Trining } & \multirow{2}{*}{\multicolumn{2}{|c|}{ Total }} \\
\hline & & \multicolumn{2}{|c|}{$<10$ time's } & \multicolumn{2}{|c|}{$10-20$ time's } & \multicolumn{2}{|c|}{$>20$ time's } & & \\
\hline & & $\mathrm{N}$ & $\%$ & $\mathrm{n}$ & $\%$ & $\mathrm{n}$ & $\%$ & $\mathrm{n}$ & $\%$ \\
\hline 1. & $20-25$ years & 15 & 88,2 & 2 & 11,8 & 0 & 0 & 17 & 100,0 \\
\hline 2. & $26-30$ years & 20 & 40,8 & 29 & 59,2 & 0 & 0 & 49 & 100,0 \\
\hline 3. & $>30$ years & 3 & 21,4 & 8 & 57,1 & 3 & 21,4 & 14 & 100,0 \\
\hline \multicolumn{2}{|c|}{$\frac{1}{\text { Total }}$} & 38 & 47,5 & 39 & 48,8 & 3 & 3,8 & 80 & 100,0 \\
\hline
\end{tabular}

c. Analysis Years of Service Nurse's Of Education of Nurse's

Table 5.46 Cross Tabulation Years of Service With Education of Nurse's

\begin{tabular}{|c|c|c|c|c|c|c|c|}
\hline \multirow{3}{*}{ No } & \multirow{3}{*}{$\begin{array}{l}\text { Years of } \\
\text { Service }\end{array}$} & \multicolumn{4}{|c|}{ Education of } & \multirow{2}{*}{\multicolumn{2}{|c|}{ Total }} \\
\hline & & \multicolumn{2}{|c|}{ Bachelor } & \multicolumn{2}{|c|}{ Diploma } & & \\
\hline & & $\mathrm{N}$ & $\%$ & $\mathrm{n}$ & $\%$ & $\mathrm{n}$ & $\%$ \\
\hline 1. & $0-5$ years & 14 & 27,5 & 37 & 72,5 & 51 & 100,0 \\
\hline 2. & $6-10$ years & 2 & 8,3 & 22 & 91,7 & 24 & 100,0 \\
\hline 3. & $>10$ years & 0 & 0 & 5 & 100,0 & 5 & 100,0 \\
\hline \multicolumn{2}{|c|}{$\frac{1}{\text { Total }}$} & 42 & 52,5 & 38 & 47,5 & 80 & 100,0 \\
\hline
\end{tabular}


d. Analysis Years of Service Nurse's Of Trining Nurse's

Table 5.47 Cross Tabulation Years of Service With Trining Nurse's

\begin{tabular}{|c|c|c|c|c|c|c|c|c|c|}
\hline \multirow{3}{*}{ No } & \multirow{3}{*}{ Years of Service } & \multicolumn{6}{|c|}{ Trining } & \multirow{2}{*}{\multicolumn{2}{|c|}{ Total }} \\
\hline & & \multicolumn{2}{|c|}{$<10$ time's } & \multicolumn{2}{|c|}{$10-20$ time's } & \multicolumn{2}{|c|}{$>20$ time's } & & \\
\hline & & $\mathrm{n}$ & $\%$ & $\mathrm{n}$ & $\%$ & $\mathrm{n}$ & $\%$ & $\mathrm{n}$ & $\%$ \\
\hline 1. & $0-5$ years & 34 & 66,7 & 17 & 33,3 & 0 & 0 & 51 & 100,0 \\
\hline 2. & $6-10$ years & 3 & 12,5 & 20 & 83,3 & 1 & 4,2 & 24 & 100,0 \\
\hline 3. & $>10$ years & 1 & 20,0 & 2 & 40,0 & 2 & 40,0 & 5 & 100,0 \\
\hline \multicolumn{2}{|c|}{ Total } & 38 & 47,5 & 39 & 48,8 & 3 & 3,8 & 80 & 100,0 \\
\hline
\end{tabular}

2. Analysis of Individual Characteristics Hard Skill Nurse's

a. Analysis Age's Nurse's Of Hard Skill Nurse's

Table 5.48 Cross Tabulation Age's With Hard Skill Nurse's

\begin{tabular}{|c|c|c|c|c|c|c|c|}
\hline \multirow{3}{*}{ No } & \multirow{3}{*}{ Age's } & \multicolumn{4}{|c|}{ Categories Hard Skill } & \multirow{2}{*}{\multicolumn{2}{|c|}{ Total }} \\
\hline & & \multicolumn{2}{|c|}{$\begin{array}{l}\text { Memenuhi } \\
\text { Standar }\end{array}$} & \multicolumn{2}{|c|}{$\begin{array}{l}\text { Not Memenuhi } \\
\text { Standar }\end{array}$} & & \\
\hline & & $\mathrm{n}$ & $\%$ & $\mathrm{n}$ & $\%$ & $\mathrm{n}$ & $\%$ \\
\hline 1. & $20-25$ years & 17 & 100,0 & 0 & 0 & 17 & 100,0 \\
\hline 2. & $26-30$ years & 47 & 95,9 & 2 & 4,1 & 49 & 100,0 \\
\hline 3. & $>30$ years & 10 & 71,4 & 4 & 28,6 & 14 & 100,0 \\
\hline \multicolumn{2}{|c|}{ Total } & 74 & 92,5 & 6 & 7,5 & 80 & 100,0 \\
\hline
\end{tabular}

b. Analysis Masa Kerja Of Hard Skill Nurse's Buleleng

Table 5.49 Cross TabulationYears of Service With Hard Skill Nurse's

\begin{tabular}{|c|c|c|c|c|c|c|c|}
\hline \multirow{3}{*}{ No } & \multirow{3}{*}{$\begin{array}{l}\text { Years of } \\
\text { Service }\end{array}$} & \multicolumn{4}{|c|}{ Categories Hard Skill } & \multirow{2}{*}{\multicolumn{2}{|c|}{ Total }} \\
\hline & & \multicolumn{2}{|c|}{$\begin{array}{l}\text { Memenuhi } \\
\text { Standar }\end{array}$} & \multicolumn{2}{|c|}{$\begin{array}{l}\text { Not Memenuhi } \\
\text { Standar }\end{array}$} & & \\
\hline & & $\mathrm{n}$ & $\%$ & $\mathrm{n}$ & $\%$ & $\mathrm{n}$ & $\%$ \\
\hline 1. & $0-5$ years & 50 & 98,0 & 1 & 2,0 & 51 & 100,0 \\
\hline 2. & $6-10$ years & 22 & 91,7 & 2 & 8,3 & 24 & 100,0 \\
\hline 3. & $>10$ years & 2 & 40,0 & 3 & 60,0 & 5 & 100,0 \\
\hline \multicolumn{2}{|c|}{ Total } & 74 & 92,5 & 6 & 7,5 & 80 & 100,0 \\
\hline
\end{tabular}

3. Analysis of Individual Characteristics Soft Skill Nurse's

a. Analysis Age's Nurse's Of Soft Skill Nurse's

Table 5.50 Cross Tabulation Age's With Soft Skill Nurse's 5

\begin{tabular}{|c|c|c|c|c|c|c|c|}
\hline \multirow{3}{*}{ No } & \multirow{3}{*}{ Age's } & \multicolumn{4}{|c|}{ Categories Soft Skill } & \multirow{2}{*}{\multicolumn{2}{|c|}{ Total }} \\
\hline & & \multicolumn{2}{|c|}{ Good } & \multicolumn{2}{|c|}{$\mathrm{Bad}$} & & \\
\hline & & $\mathrm{n}$ & $\%$ & $\mathrm{n}$ & $\%$ & $\mathrm{n}$ & $\%$ \\
\hline 1. & $20-25$ years & 2 & 11,8 & 15 & 88,2 & 17 & 100,0 \\
\hline 2. & $26-30$ years & 10 & 20,4 & 39 & 79,6 & 49 & 100,0 \\
\hline 3. & $>30$ years & 4 & 28,6 & 10 & 71,4 & 14 & 100,0 \\
\hline \multicolumn{2}{|c|}{ Total } & 16 & 20,0 & 64 & 80,0 & 80 & 100,0 \\
\hline
\end{tabular}


b. Analysis Years of Service Of Soft Skill Nurse's

Table 5.51 Cross TabulationYears of Service With Soft Skill Nurse's

\begin{tabular}{|c|c|c|c|c|c|c|c|}
\hline \multirow{3}{*}{ No } & \multirow{3}{*}{$\begin{array}{l}\text { Years of } \\
\text { Service }\end{array}$} & \multicolumn{4}{|c|}{ Categories Soft Skill } & \multirow{2}{*}{\multicolumn{2}{|c|}{ Total }} \\
\hline & & \multicolumn{2}{|c|}{ Good } & \multicolumn{2}{|c|}{$\mathrm{Bad}$} & & \\
\hline & & $\mathrm{n}$ & $\%$ & $\mathrm{n}$ & $\%$ & $\mathrm{n}$ & $\%$ \\
\hline 1. & $0-5$ years & 8 & 15,7 & 43 & 84,3 & 51 & 100,0 \\
\hline 2. & $6-10$ years & 8 & 33,3 & 16 & 66,7 & 24 & 100,0 \\
\hline 3. & $>10$ years & 0 & 0 & 5 & 100,0 & 5 & 100,0 \\
\hline \multicolumn{2}{|c|}{ Total } & 16 & 20,0 & 64 & 80,0 & 80 & 100,0 \\
\hline
\end{tabular}

c. Analysis Extra IncomeOf Soft Skill Nurse's

Table 5.52 Cross Tabulation Extra Income With Soft Skill

\begin{tabular}{|c|c|c|c|c|c|c|c|}
\hline \multirow{3}{*}{ No } & \multirow{3}{*}{ Extra Income } & \multicolumn{4}{|c|}{ Categories Soft Skill } & \multirow{2}{*}{\multicolumn{2}{|c|}{ Total }} \\
\hline & & \multicolumn{2}{|c|}{ Good } & \multicolumn{2}{|c|}{$\mathrm{Bad}$} & & \\
\hline & & $\mathrm{n}$ & $\%$ & $\mathrm{n}$ & $\%$ & $\mathrm{n}$ & $\%$ \\
\hline 1. & $<\mathrm{Rp} 3.000 .000$ & 3 & 10,0 & 27 & 90,0 & 30 & 100,0 \\
\hline 2. & Rp 3.000.000 - Rp 5.999.999 & 11 & 24,4 & 24 & 75,6 & 45 & 100,0 \\
\hline 3. & Rp 6.000.000 - Rp 8.999.999 & 1 & 33,3 & 2 & 66,7 & 3 & 100,0 \\
\hline 4. & $\geq \operatorname{Rp} 9.000 .000$ & 1 & 50,0 & 1 & 50,0 & 2 & 100,0 \\
\hline \multicolumn{2}{|c|}{ Total } & 16 & 20,0 & 64 & 80,0 & 80 & 100,0 \\
\hline
\end{tabular}

4. Analysis of Individual Characteristics Work Ethics Nurse's

a. Analysis Age's Nurse's Of Work Ethics Nurse's

Table 5.53 Cross Tabulation Age's With Work Ethics Nurse's

\begin{tabular}{|c|c|c|c|c|c|c|c|}
\hline \multirow{3}{*}{ No } & \multirow{3}{*}{ Age's } & \multicolumn{4}{|c|}{ Categories Work Ethics } & \multirow{2}{*}{\multicolumn{2}{|c|}{ Total }} \\
\hline & & \multicolumn{2}{|c|}{ Good } & \multicolumn{2}{|c|}{$\mathrm{Bad}$} & & \\
\hline & & $\mathrm{n}$ & $\%$ & $\mathrm{n}$ & $\%$ & $n$ & $\%$ \\
\hline 1. & $20-25$ years & 0 & 0 & 17 & 100,0 & 17 & 100,0 \\
\hline 2. & $26-30$ years & 7 & 14,3 & 42 & 85,7 & 49 & 100,0 \\
\hline 3. & $>30$ years & 0 & 0 & 14 & 100,0 & 14 & 100,0 \\
\hline \multicolumn{2}{|c|}{ Total } & 7 & 8,8 & 73 & 91,3 & 80 & 100,0 \\
\hline
\end{tabular}

b. Analysis Years of Service Of Work Ethics Nurse's

Table 5.54 Cross Tabulation Years of Service With Work Ethics Nurse's

\begin{tabular}{|c|c|c|c|c|c|c|c|}
\hline \multirow{3}{*}{ No } & \multirow{3}{*}{$\begin{array}{l}\text { Years of } \\
\text { Service }\end{array}$} & \multicolumn{4}{|c|}{ Categories Work Ethics } & \multirow{2}{*}{\multicolumn{2}{|c|}{ Total }} \\
\hline & & \multicolumn{2}{|c|}{ Good } & \multicolumn{2}{|c|}{$\mathrm{Bad}$} & & \\
\hline & & $\mathrm{n}$ & $\%$ & $\mathrm{n}$ & $\%$ & $\mathrm{n}$ & $\%$ \\
\hline 1. & $0-5$ years & 5 & 9,8 & 46 & 90,2 & 51 & 100,0 \\
\hline 2. & $6-10$ years & 2 & 8,3 & 22 & 91,7 & 24 & 100,0 \\
\hline 3. & $>10$ years & 0 & 0 & 5 & 100,0 & 5 & 100,0 \\
\hline \multicolumn{2}{|c|}{ Total } & 7 & 8,8 & 73 & 91,3 & 80 & 100,0 \\
\hline
\end{tabular}


c. Analysis Extra IncomeOf Work Ethics Nurse's

Table 5.55 Cross Tabulation Extra Income With Work Ethics

\begin{tabular}{|c|c|c|c|c|c|c|c|}
\hline \multirow{3}{*}{ No } & \multirow{3}{*}{ Extra Income } & \multicolumn{4}{|c|}{ Categories Work Ethics } & \multirow{2}{*}{\multicolumn{2}{|c|}{ Total }} \\
\hline & & \multicolumn{2}{|c|}{ Good } & \multicolumn{2}{|c|}{$\mathrm{Bad}$} & & \\
\hline & & $\mathrm{n}$ & $\%$ & $\mathrm{n}$ & $\%$ & $\mathrm{n}$ & $\%$ \\
\hline 1. & $<\mathrm{Rp} 3.000 .000$ & 4 & 13,3 & 26 & 86,7 & 30 & 100,0 \\
\hline 2. & Rp 3.000.000 - Rp 5.999.999 & 3 & 6,7 & 42 & 93,3 & 45 & 100,0 \\
\hline 3. & Rp 6.000.000 - Rp 8.999.999 & 0 & 0 & 3 & 100,0 & 3 & 100,0 \\
\hline 4. & $\geq \operatorname{Rp} 9.000 .000$ & 0 & 0 & 2 & 100,0 & 2 & 100,0 \\
\hline \multicolumn{2}{|c|}{ Total } & 7 & 8,8 & 73 & 91,3 & 80 & 100,0 \\
\hline
\end{tabular}

5. Analysis of Individual Characteristics Legal Aspect Nurse's

a. Analysis Age's Nurse's Of Nurses Competence Record

Table 5.56 Cross Tabulation Age's With Nurses Competence Record

\begin{tabular}{|c|c|c|c|c|c|c|c|}
\hline \multirow{3}{*}{ No } & \multirow{3}{*}{ Age's } & \multicolumn{4}{|c|}{ Categories Competence Result } & \multirow{2}{*}{\multicolumn{2}{|c|}{ Total }} \\
\hline & & \multicolumn{2}{|c|}{ One Times exam } & \multicolumn{2}{|c|}{ Two Times exam } & & \\
\hline & & $\mathrm{n}$ & $\%$ & $\mathrm{n}$ & $\%$ & $\mathrm{n}$ & $\%$ \\
\hline 1. & $20-25$ years & 17 & 100,0 & 0 & 0 & 17 & 100,0 \\
\hline 2. & $26-30$ years & 48 & 98,0 & 1 & 2,0 & 49 & 100,0 \\
\hline 3. & $>30$ years & 14 & 100,0 & 0 & 0 & 14 & 100,0 \\
\hline \multicolumn{2}{|c|}{ Total } & 79 & 98,8 & 1 & 1,3 & 80 & 100,0 \\
\hline
\end{tabular}

b. Analysis Age's Nurse's Of Nurse's Registration Certificate(STR) Nurse's

Table 5.57 Cross Tabulation Age's With Nurse's Registration Certificate (STR) Nurse's

\begin{tabular}{|c|c|c|c|c|c|c|c|}
\hline \multirow{3}{*}{ No } & \multirow{3}{*}{ Age's } & \multicolumn{4}{|c|}{ Categories STR } & \multirow{2}{*}{\multicolumn{2}{|c|}{ Total }} \\
\hline & & \multicolumn{2}{|c|}{$\begin{array}{l}\text { STR Masih } \\
\text { Berlaku }\end{array}$} & \multicolumn{2}{|c|}{$\begin{array}{c}\text { STR Habis Masa } \\
\text { Berlaku }\end{array}$} & & \\
\hline & & $\mathrm{n}$ & $\%$ & $\mathrm{n}$ & $\%$ & $\mathrm{~N}$ & $\%$ \\
\hline 1. & $20-25$ years & 17 & 100,0 & 0 & 0,0 & 17 & 100,0 \\
\hline 2. & $26-30$ years & 42 & 85,7 & 7 & 14,3 & 49 & 100,0 \\
\hline 3. & $>30$ years & 13 & 92,9 & 1 & 7,1 & 14 & 100,0 \\
\hline \multicolumn{2}{|c|}{ Total } & 72 & 90,0 & 8 & 10,0 & 80 & 100,0 \\
\hline
\end{tabular}

c. Analysis Age's Nurse's Of Nurse's Practic License(SIP) Nurse's

Table 5.58 Cross Tabulation Age's With Nurse's Practic License(SIP) Nurse's

\begin{tabular}{|l|l|r|r|r|r|r|r|}
\hline \multirow{2}{*}{ No } & \multirow{2}{*}{ Age's } & \multicolumn{4}{|c|}{ Categories SIP } & \multicolumn{2}{c|}{ Total } \\
\cline { 3 - 7 } & & \multicolumn{2}{|c|}{$\begin{array}{c}\text { SIP Masih } \\
\text { Berlaku }\end{array}$} & \multicolumn{2}{c|}{$\begin{array}{c}\text { SIP Habis Masa } \\
\text { Berlaku }\end{array}$} & \multicolumn{2}{c|}{} \\
\cline { 3 - 8 } & & $\mathrm{n}$ & $\%$ & $\mathrm{~N}$ & $\%$ & $\mathrm{~N}$ & $\%$ \\
\hline 1. & $20-25$ years & 17 & 100,0 & 0 & 0,0 & 17 & 100,0 \\
\hline 2. & $26-30$ years & 42 & 85,7 & 7 & 14,3 & 49 & 100,0 \\
\hline 3. & $>30$ years & 13 & 92,9 & 1 & 7,1 & 14 & 100,0 \\
\hline \multicolumn{2}{|l|}{ Total } & 39 & 72 & 90,0 & 8 & 10,0 & 80 \\
\hline
\end{tabular}


d. Analysis Years of Service Of Nurses Competence Record

Table 5.59 Cross Tabulation Years of Service With Nurses Competence Record

\begin{tabular}{|c|c|c|c|c|c|c|c|}
\hline \multirow{3}{*}{ No } & \multirow{3}{*}{$\begin{array}{l}\text { Years of } \\
\text { Service }\end{array}$} & \multicolumn{4}{|c|}{ Categories Competency Test Results } & \multirow{2}{*}{\multicolumn{2}{|c|}{ Total }} \\
\hline & & \multicolumn{2}{|c|}{ One Times exam } & \multicolumn{2}{|c|}{ Two Times exam } & & \\
\hline & & $\mathrm{n}$ & $\%$ & $\mathrm{n}$ & $\%$ & $\mathrm{~N}$ & $\%$ \\
\hline 1. & $0-5$ years & 51 & 100,0 & 0 & 0 & 51 & 100,0 \\
\hline 2. & $6-10$ years & 24 & 100,0 & 0 & 0 & 24 & 100,0 \\
\hline 3. & $>10$ years & 4 & 80,0 & 1 & 20,0 & 5 & 100,0 \\
\hline \multicolumn{2}{|c|}{ Total } & 79 & 98,8 & 1 & 1,2 & 80 & 100,0 \\
\hline
\end{tabular}

e. Analysis Years of Service Of Nurse's Registration Certificate (STR) Nurse's

Table 5.60 Cross Tabulation Years of Service With Nurse's Registration Certificate(STR) Nurse's

\begin{tabular}{|l|l|r|r|r|r|r|r|}
\hline \multirow{2}{*}{ No } & \multirow{2}{*}{$\begin{array}{l}\text { Years of } \\
\text { Service }\end{array}$} & \multicolumn{4}{|c|}{$\begin{array}{c}\text { Categories STR } \\
\text { STR Masih } \\
\text { Berlaku }\end{array}$} & $\begin{array}{c}\text { STR Habis Masa } \\
\text { Berlaku }\end{array}$ & \multicolumn{2}{|c|}{ Total } \\
\cline { 3 - 7 } & & $\mathrm{n}$ & \multicolumn{1}{|c|}{$\%$} & $\mathrm{n}$ & \multicolumn{1}{c|}{$\%$} & $\mathrm{n}$ & $\%$ \\
\hline 1. & $0-5$ years & 43 & 84,2 & 8 & 15,7 & 51 & 100,0 \\
\hline 2. & $6-10$ years & 24 & 100,0 & 0 & 0,0 & 24 & 100,0 \\
\hline 3. & $>10$ years & 5 & 100.0 & 0 & 0,0 & 5 & 100,0 \\
\hline \multicolumn{2}{|l|}{ Total } & 72 & 90,0 & 8 & 10,0 & 80 & 100,0 \\
\hline
\end{tabular}

f. Analysis Years of Service Of Nurse's Practic License (SIP) Nurse's

Table 5.61 Cross Tabulation Years of Service With Nurse's Practic License(SIP) Nurse's

\begin{tabular}{|c|c|c|c|c|c|c|c|}
\hline \multirow{3}{*}{ No } & \multirow{3}{*}{$\begin{array}{l}\text { Years of } \\
\text { Service }\end{array}$} & \multicolumn{4}{|c|}{ Categories SIP } & \multirow{2}{*}{\multicolumn{2}{|c|}{ Total }} \\
\hline & & \multicolumn{2}{|c|}{$\begin{array}{l}\text { SIP Still } \\
\text { Valid }\end{array}$} & \multicolumn{2}{|c|}{ SIP Expired } & & \\
\hline & & $\mathrm{n}$ & $\%$ & $\mathrm{~N}$ & $\%$ & $\mathrm{n}$ & $\%$ \\
\hline 1. & $0-5$ years & 43 & 84,2 & 8 & 15,7 & 51 & 100,0 \\
\hline 2. & $6-10$ years & 24 & 100,0 & 0 & 0,0 & 24 & 100,0 \\
\hline 3. & $>10$ years & 5 & 100.0 & 0 & 0,0 & 5 & 100,0 \\
\hline \multicolumn{2}{|c|}{$\frac{1}{\text { Total }}$} & 39 & 72 & 90,0 & 8 & 10,0 & 80 \\
\hline
\end{tabular}

H. Analysis of Individual Characteristics Service Quality Nurse's

1. Analysis Age's Nurse's Of Service Quality Nurse's

Table 5.62 Cross Tabulation Age's With Service Quality Nurse's

\begin{tabular}{|c|c|c|c|c|c|c|c|}
\hline \multirow{3}{*}{ No } & \multirow{3}{*}{ Age's } & \multicolumn{4}{|c|}{ Categories Service Quality } & & \\
\hline & & \multicolumn{2}{|c|}{$\begin{array}{l}\text { Service Quality } \\
\text { Good }\end{array}$} & \multicolumn{2}{|c|}{$\begin{array}{c}\text { Service Quality } \\
\text { Bad }\end{array}$} & \multicolumn{2}{|c|}{ Total } \\
\hline & & $\mathrm{n}$ & $\%$ & $\mathrm{~N}$ & $\%$ & $\mathrm{n}$ & $\%$ \\
\hline 1. & $20-25$ years & 7 & 41,2 & 10 & 58,8 & 17 & 100,0 \\
\hline 2. & $26-30$ years & 28 & 57,1 & 21 & 42,9 & 49 & 100,0 \\
\hline 3. & $>30$ years & 7 & 50,0 & 7 & 50,0 & 14 & 100,0 \\
\hline \multicolumn{2}{|c|}{ Total } & 42 & 52,5 & 38 & 47,5 & 80 & 100,0 \\
\hline
\end{tabular}


2. Analysis Years of Service Of Service Quality Nurse's

Table 5.63 Cross TabulationYears of Service With Service Quality Nurse's

\begin{tabular}{|c|c|c|c|c|c|c|c|}
\hline \multirow{3}{*}{ No } & \multirow{3}{*}{$\begin{array}{l}\text { Years of } \\
\text { Service }\end{array}$} & \multicolumn{4}{|c|}{ Categories Service Quality } & & \\
\hline & & \multicolumn{2}{|c|}{$\begin{array}{c}\text { Service Quality } \\
\text { Good }\end{array}$} & \multicolumn{2}{|c|}{$\begin{array}{c}\text { Service Quality } \\
\text { Bad }\end{array}$} & \multicolumn{2}{|c|}{ Total } \\
\hline & & $\mathrm{n}$ & $\%$ & $\mathrm{~N}$ & $\%$ & $\mathrm{n}$ & $\%$ \\
\hline 1. & $0-5$ years & 26 & 52,0 & 25 & 49,0 & 51 & 100,0 \\
\hline 2. & $6-10$ years & 12 & 50,0 & 12 & 50,0 & 24 & 100,0 \\
\hline 3. & $>10$ years & 4 & 80,0 & 1 & 20,0 & 5 & 100,0 \\
\hline \multicolumn{2}{|c|}{ Total } & 42 & 52,5 & 38 & 47,5 & 80 & 100,0 \\
\hline
\end{tabular}

3. Analysis Extra IncomeOf Service Quality Nurse's

Table 5.64 Cross TabulationExtra Income With Service Quality Nurse's

\begin{tabular}{|c|c|c|c|c|c|c|c|}
\hline \multirow{3}{*}{ No } & \multirow{3}{*}{ Extra Income } & \multicolumn{4}{|c|}{ Categories Service Quality } & \multirow{2}{*}{\multicolumn{2}{|c|}{ Total }} \\
\hline & & \multicolumn{2}{|c|}{$\begin{array}{c}\text { Service Quality } \\
\text { Goo }\end{array}$} & \multicolumn{2}{|c|}{$\begin{array}{c}\text { Service Quality } \\
\text { Bad }\end{array}$} & & \\
\hline & & $\mathrm{n}$ & $\%$ & $\mathrm{n}$ & $\%$ & $\mathrm{n}$ & $\%$ \\
\hline 1. & $<\operatorname{Rp} 3.000 .000$ & 16 & 53,3 & 14 & 46,7 & 30 & 100,0 \\
\hline 2. & $\operatorname{Rp} 3.000 .000-\operatorname{Rp} 5.999 .999$ & 24 & 53,3 & 21 & 46,7 & 45 & 100,0 \\
\hline 3. & Rp 6.000.000 - Rp 8.999.999 & 2 & 66,7 & 1 & 33,3 & 3 & 0,0 \\
\hline 4. & $\geq \operatorname{Rp} 9.000 .000$ & 0 & 0,0 & 2 & 100,0 & 2 & 100,0 \\
\hline Tot & & 42 & 52,5 & 38 & 47,5 & 80 & 100,0 \\
\hline
\end{tabular}

4. Analysis Position Head of FamilyWith Service Quality Nurse's

Table 5.65 Cross TabulationPosition Head of FamilyWith Service Quality Nurse's

\begin{tabular}{|c|c|c|c|c|c|c|c|}
\hline \multirow{3}{*}{ No } & \multirow{3}{*}{ Head Family Position } & \multicolumn{4}{|c|}{ Categories Service Quality } & \multirow{2}{*}{\multicolumn{2}{|c|}{ Total }} \\
\hline & & \multicolumn{2}{|c|}{$\begin{array}{c}\text { Kualitas } \\
\text { Layanan Good }\end{array}$} & \multicolumn{2}{|c|}{$\begin{array}{c}\text { Kualitas } \\
\text { Layanan Bad }\end{array}$} & & \\
\hline & & $\mathrm{n}$ & $\%$ & $\mathrm{n}$ & $\%$ & $\mathrm{n}$ & $\%$ \\
\hline 1. & No Having Position & 13 & 50,0 & 13 & 50,0 & 26 & 100,0 \\
\hline 2. & Staff Level & 27 & 55,1 & 22 & 44,9 & 49 & 100,0 \\
\hline 3. & Unit Head Level & 2 & 40,0 & 3 & 60,0 & 5 & 100,0 \\
\hline \multicolumn{2}{|c|}{ Total } & 31 & 52,5 & 28 & 47,5 & 59 & 100,0 \\
\hline
\end{tabular}

L. Analysis Nurse's C om p e t e n e Of Service Quality Nurse's

1. Analysis Basic Knowledge Nurse's With Service Quality Nurse's

Table 5.66 Cross Tabulation Education of With Service Quality Nurse's

\begin{tabular}{|c|c|c|c|c|c|c|c|}
\hline \multirow{3}{*}{ No } & \multirow{3}{*}{ Education of } & \multicolumn{4}{|c|}{ Categories Service Quality } & & \\
\hline & & \multicolumn{2}{|c|}{$\begin{array}{c}\text { Service Quality } \\
\text { Good }\end{array}$} & \multicolumn{2}{|c|}{$\begin{array}{c}\text { Kualitas } \\
\text { Layanan Bad }\end{array}$} & \multicolumn{2}{|c|}{ Total } \\
\hline & & $\mathrm{n}$ & $\%$ & $\mathrm{n}$ & $\%$ & $\mathrm{n}$ & $\%$ \\
\hline 1. & Diploma III & 36 & 56,3 & 28 & 43,8 & 64 & 100,0 \\
\hline 2. & Bachelor & 6 & 37,5 & 10 & 62,5 & 16 & 100,0 \\
\hline \multicolumn{2}{|c|}{ Total } & 42 & 52,5 & 38 & 47,5 & 80 & 100,0 \\
\hline
\end{tabular}


2. Analysis Trining Nurse's Of Kualitas Layanan Nurse's Buleleng Table 5.67 Cross Tabulation Trining With Service Quality Nurse's

\begin{tabular}{|l|l|r|r|r|r|r|r|}
\hline \multirow{2}{*}{ No } & \multirow{2}{*}{ Categories Trining } & \multicolumn{4}{|c|}{ Categories Service Quality } & \multicolumn{2}{|c|}{} \\
\cline { 3 - 8 } & & \multicolumn{2}{|c|}{$\begin{array}{c}\text { Service Quality } \\
\text { Good }\end{array}$} & \multicolumn{2}{c|}{$\begin{array}{c}\text { Kualitas } \\
\text { Layanan Bad }\end{array}$} & \multicolumn{2}{|c|}{ Total } \\
\cline { 3 - 8 } & & $\mathrm{n}$ & $\%$ & $\mathrm{n}$ & \multicolumn{1}{c|}{$\mathrm{n}$} & $\%$ \\
\hline 1. & $<10$ time's & 20 & 52,6 & 18 & 47,4 & 38 & 100,0 \\
\hline 2. & $10-20$ time's & 19 & 48,7 & 20 & 51,3 & 39 & 100,0 \\
\hline 3. & $>20$ time's & 3 & 100,0 & 0 & 0,0 & 3 & 100,0 \\
\hline \multicolumn{2}{|l|}{ Total } & 42 & 52,5 & 38 & 47,5 & 80 & 100,0 \\
\hline
\end{tabular}

2 Analysis Hard Skill Of Service Quality Nurse's

Table 5.68 Cross Tabulation Hard Skill With Service Quality Nurse's

\begin{tabular}{|c|c|c|c|c|c|c|c|}
\hline \multirow{3}{*}{ No } & \multirow{3}{*}{ Categories Hard Skill } & \multicolumn{4}{|c|}{ Categories Service Quality } & \multirow{2}{*}{\multicolumn{2}{|c|}{ Total }} \\
\hline & & \multicolumn{2}{|c|}{$\begin{array}{l}\text { Kualitas } \\
\text { Lavanan }\end{array}$} & \multicolumn{2}{|c|}{$\begin{array}{c}\text { Kualitas } \\
\text { Lavanan Bad }\end{array}$} & & \\
\hline & & $\mathrm{n}$ & $\%$ & $\mathrm{n}$ & $\%$ & $\mathrm{n}$ & $\%$ \\
\hline 1. & Hard Skill Meet Standards & 39 & 52,7 & 35 & 47,3 & 74 & 100,0 \\
\hline 2. & Hard Skill Not Meeting the & 3 & 50,0 & 3 & 50,0 & 6 & 100,0 \\
\hline Tot: & & 42 & 52,5 & 38 & 47,5 & 80 & 100,0 \\
\hline
\end{tabular}

3. Analysis Soft Skill Nurse's Of Service Quality Nurse's

Table 5.69 Cross Tabulation Soft Skill With Service Quality Nurse's

\begin{tabular}{|c|c|c|c|c|c|c|c|}
\hline \multirow{3}{*}{ No } & \multirow{3}{*}{ Categories Soft Skill } & \multicolumn{4}{|c|}{ Categories Service Quality } & \multirow{2}{*}{\multicolumn{2}{|c|}{ Total }} \\
\hline & & \multicolumn{2}{|c|}{$\begin{array}{c}\text { Service Quality } \\
\text { Good }\end{array}$} & \multicolumn{2}{|c|}{$\begin{array}{c}\text { Kualitas } \\
\text { Layanan Bad }\end{array}$} & & \\
\hline & & $\mathrm{n}$ & $\%$ & $\mathrm{n}$ & $\%$ & $\mathrm{n}$ & $\%$ \\
\hline 1. & Soft Skill Good & 13 & 81,3 & 3 & 18,7 & 16 & 100,0 \\
\hline 2. & Soft Skill Bad & 29 & 45,3 & 35 & 54,7 & 64 & 100,0 \\
\hline \multicolumn{2}{|c|}{ Total } & 42 & 52,5 & 38 & 47,5 & 80 & 100,0 \\
\hline
\end{tabular}

4. Analysis Work Ethics Nurse's Of Service Quality Nurse's

Table 5.70 Cross Tabulation Work Ethics With Service Quality Nurse's

\begin{tabular}{|c|c|c|c|c|c|c|c|}
\hline \multirow{3}{*}{ No } & \multirow{3}{*}{ Categories Work Ethics } & \multicolumn{4}{|c|}{ Categories Service Quality } & \multirow{2}{*}{\multicolumn{2}{|c|}{ Total }} \\
\hline & & \multicolumn{2}{|c|}{$\begin{array}{c}\text { Good Service } \\
\text { Quality }\end{array}$} & \multicolumn{2}{|c|}{$\begin{array}{c}\text { Bad Service } \\
\text { Quality }\end{array}$} & & \\
\hline & & $\mathrm{n}$ & $\%$ & $\mathrm{n}$ & $\%$ & $\mathrm{n}$ & $\%$ \\
\hline 1. & Work Ethics Good & 3 & 42,9 & 7 & 57,1 & 7 & 100,0 \\
\hline 2. & Work Ethics Bad & 39 & 53,4 & 34 & 46,6 & 73 & 100,0 \\
\hline \multicolumn{2}{|c|}{ Total } & 42 & 52,5 & 38 & 47,5 & 80 & 100,0 \\
\hline
\end{tabular}


5. Analysis Legal Aspect Nurse's Of Service Quality Nurse's

a Analysis Competency Test ResultsOf Service Quality Nurse's

Table 5.71 Cross Tabulation Competency Test Results With Service Quality Nurse's

\begin{tabular}{|c|c|c|c|c|c|c|c|}
\hline \multirow{3}{*}{ No } & \multirow{3}{*}{$\begin{array}{l}\text { Result exam } \\
\text { Categories }\end{array}$} & \multicolumn{4}{|c|}{ Categories Service Quality } & \multirow{2}{*}{\multicolumn{2}{|c|}{ Total }} \\
\hline & & \multicolumn{2}{|c|}{$\begin{array}{c}\text { Good Service } \\
\text { Quality }\end{array}$} & \multicolumn{2}{|c|}{$\begin{array}{c}\text { Bad Service } \\
\text { Quality }\end{array}$} & & \\
\hline & & $\mathrm{n}$ & $\%$ & $\mathrm{~N}$ & $\%$ & $\mathrm{n}$ & $\%$ \\
\hline 1. & One Times exam & 41 & 51,9 & 38 & 48,1 & 79 & 100,0 \\
\hline 2. & Two Times exam & 1 & 100,0 & 0 & $\overline{0,0}$ & 1 & 100,0 \\
\hline \multicolumn{2}{|c|}{ Total } & 42 & 52,5 & 38 & 47,5 & 80 & 100,0 \\
\hline
\end{tabular}

b. Analysis Nurse's Registration Certificate(STR) Of Service Quality Nurse's

Table 5.72 Cross TabulationNurse's Registration Certificate(STR) With Service Quality Nurse's

\begin{tabular}{|c|c|c|c|c|c|c|c|}
\hline \multirow{3}{*}{ No } & \multirow{3}{*}{$\begin{array}{c}\text { Categories Letter of } \\
\text { Sign Registration } \\
\text { (STR) }\end{array}$} & \multicolumn{4}{|c|}{ Categories Service Quality } & \multirow{2}{*}{\multicolumn{2}{|c|}{ Total }} \\
\hline & & \multicolumn{2}{|c|}{ Good } & \multicolumn{2}{|c|}{$\mathrm{Bad}$} & & \\
\hline & & $\mathrm{n}$ & $\%$ & $\mathrm{~N}$ & $\%$ & $\mathrm{n}$ & $\%$ \\
\hline 1. & STR Still Valid & 18 & 46,2 & 21 & 53,8 & 39 & 100,0 \\
\hline 2. & STR Expiry date & 24 & 58,5 & 17 & 41,5 & 41 & 100,0 \\
\hline \multicolumn{2}{|c|}{ Total } & 42 & 52,5 & 38 & 47,5 & 80 & 100,0 \\
\hline
\end{tabular}

c. Analysis Nurse's Practic License(SIP) Of Service Quality Nurse's

Table 5.73 Cross TabulationNurse's Practic License(SIP) With Service Quality Nurse's

\begin{tabular}{|c|c|c|c|c|c|c|c|}
\hline \multirow{3}{*}{ No } & \multirow{3}{*}{$\begin{array}{c}\text { Categories Letter } \\
\text { License } \\
\text { (SIP) }\end{array}$} & \multicolumn{4}{|c|}{ Categories Service Quality } & \multirow{2}{*}{\multicolumn{2}{|c|}{ Total }} \\
\hline & & \multicolumn{2}{|c|}{ Good } & \multicolumn{2}{|c|}{$\mathrm{Bad}$} & & \\
\hline & & $\mathrm{n}$ & $\%$ & $\mathrm{~N}$ & $\%$ & $\mathrm{n}$ & $\%$ \\
\hline 1. & SIP Still Valid & 18 & 46,2 & 21 & 53,8 & 39 & 100,0 \\
\hline 2. & SIP Expiry date & 24 & 58,5 & 17 & 41,5 & 41 & 100,0 \\
\hline \multicolumn{2}{|c|}{ Total } & 42 & 52,5 & 38 & 47,5 & 80 & 100,0 \\
\hline
\end{tabular}

M. Analysis The Effect of Individual Characteristics of Nurse's Competence in Inpatient Installation

1. Analysis the Effect of Individual Of Education of Nurse's

Table 5.74 Summary of Statistic Logistik Multinomial Regression Individual Characteristic of Education of Nurse's

\begin{tabular}{|l|l|c|c|c|l|}
\hline No & \multicolumn{1}{|c|}{ Variable' } & $\mathrm{p}$ & $\beta$ & $\mathrm{RR}$ & \multicolumn{1}{|c|}{ Information } \\
\hline 1. & Age's Nurse's & 0,956 & 0,010 & 1.010 & Not Significant \\
\hline 2. & Years of Service Nurse's & 0,015 & $-0,498$ & 0,608 & Significant \\
\hline 3. & Extra Income & 0,236 & 0,000 & 1,000 & Not Significant \\
\hline 4. & Head Family Position & 0,890 & 0,250 & 1,284 & Not Significant \\
\hline
\end{tabular}


2. Analysis of the Effects of Individual Characteristics Of Trining Nurse's

Table 5.75 Summary of Regression Logistik Multinomial Individual Characteristics of Trining Nurse's

\begin{tabular}{|l|l|c|r|c|l|}
\hline No & \multicolumn{1}{|c|}{ Variable' } & $\mathrm{p}$ & $\beta$ & $\mathrm{RR}$ & \multicolumn{1}{|c|}{ Information } \\
\hline 1. & Age's Nurse's & 0,389 & 0,130 & 1,139 & Not Significant \\
\hline 2. & Years of Service Nurse's & 0,001 & $-0,625$ & 0,535 & Significant \\
\hline 3. & Extra Income & 0,909 & 0,000 & 1,000 & Not Significant \\
\hline 4. & Head Family Position & 0,742 & $-0,593$ & 0,742 & Not Significant \\
\hline
\end{tabular}

2 Analysis Individual Characteristic Effect Of Hard Skill Nurse's In the Inpatient Instalation Table 5.76 Summary Multinomial Logistic Regression Statistical Test ResultsIndividual Characteristic of Hard Skill Nurse's

\begin{tabular}{|l|l|c|c|c|l|}
\hline No & \multicolumn{1}{|c|}{$\begin{array}{c}\text { Variable' } \\
\text { s }\end{array}$} & $\mathrm{p}$ & $\beta$ & $\mathrm{RR}$ & \multicolumn{1}{|c|}{ Information } \\
\hline 1. & Age's Nurse's & 0,068 & $-0,304$ & 0,738 & Not Significant \\
\hline 2. & Years of Service Nurse's & 0,041 & $-0,367$ & 0,693 & Significant \\
\hline 3. & Extra Income & 0,487 & 0,000 & 1,000 & Not Significant \\
\hline 4. & Head Family Position & 0,638 & 1,264 & 3,541 & Not Significant \\
\hline
\end{tabular}

3. Analysis Individual Characteristic Effect Of Soft Skill Nurse's In the Inpatient Instalation Table 5.77 Summary Multinomial Logistic Regression Statistical Test ResultsIndividual Characteristic of Soft Skill Nurse's

\begin{tabular}{|l|l|c|r|r|l|}
\hline No & \multicolumn{1}{|c|}{$\begin{array}{c}\text { Variable' } \\
\text { s }\end{array}$} & $\mathrm{p}$ & $\beta$ & $\mathrm{RR}$ & \multicolumn{1}{|c|}{ Information } \\
\hline 1. & Age's Nurse's & 0,490 & 0,082 & 1,086 & Not Significant \\
\hline 2. & Years of Service Nurse's & 0,630 & $-0,059$ & 0,942 & Not Significant \\
\hline 3. & Extra Income & 0,365 & 0,000 & 1,000 & Not Significant \\
\hline 4. & Head Family Position & 0,688 & $-0,754$ & 0,470 & Not Significant \\
\hline
\end{tabular}

4. Analysis Individual Characteristic Effect Of Work Ethics Nurse's In the Inpatient Instalation Table 5.78 Summary Statisticall Regression of Logistik Multinomial Individual Characteristic of Work Ethics Nurse's

\begin{tabular}{|l|l|r|r|r|l|}
\hline No & \multicolumn{1}{|c|}{ Variable } & \multicolumn{1}{c|}{$\mathrm{p}$} & \multicolumn{1}{c|}{$\beta$} & \multicolumn{1}{c|}{ RR } & \multicolumn{1}{|c|}{ Information } \\
\hline 1. & Age's Nurse's & 0,833 & $-0,037$ & 0,964 & Not Significant \\
\hline 2. & Years of Service Nurse's & 0,427 & $-0,162$ & 0,851 & Not Significant \\
\hline 3. & Extra Income & 0,349 & 0,000 & 1,000 & Not Significant \\
\hline 4. & Head Family Position & 0,000 & 14400 & 1794880 & Significant \\
\hline
\end{tabular}


5. Analysis Individual Characteristic Effect Of Legal Aspect Nurse's In the Inpatient Instalation

a Analysis Individual Characteristic Effect Of Nurses Competence Record

Table 5.79 Summary Multinomial Logistic Regression Statistical Test ResultsIndividual Characteristic of Nurses Competence Record

\begin{tabular}{|l|l|r|r|r|l|}
\hline No & \multicolumn{1}{|c|}{$\begin{array}{c}\text { Variable } \\
\text { 's }\end{array}$} & \multicolumn{1}{c|}{$\mathrm{p}$} & \multicolumn{1}{c|}{$\beta$} & \multicolumn{1}{c|}{ RR } & \multicolumn{1}{|c|}{ Information } \\
\hline 1. & Age's Nurse's & 0,998 & 4,668 & 106,447 & Not Significant \\
\hline 2. & Years of Service Nurse's & 0,997 & $-5,922$ & 0,851 & Not Significant \\
\hline 3. & Extra Income & 1,000 & 0,000 & 1,000 & Not Significant \\
\hline 4. & Head Family Position & 0,999 & 26,660 & 3786 E+11 & Not Significant \\
\hline
\end{tabular}

b. Analysis Individual Characteristic Effect Of Nurse's Registration Certificate(STR) Nurse's Table 5.80 Summary Multinomial Logistic Regression Statistical Test ResultsIndividual Characteristic of Registration of Nurses (STR) Nurse's

\begin{tabular}{|l|l|r|r|r|l|}
\hline No & \multicolumn{1}{|c|}{ Variable' } & \multicolumn{1}{c|}{$\mathrm{p}$} & \multicolumn{1}{c|}{$\beta$} & \multicolumn{1}{c|}{$\mathrm{RR}$} & \multicolumn{1}{c|}{ Information } \\
\hline 1. & Age's Nurse's & 0,466 & $-0,109$ & 0,897 & Not Significant \\
\hline 2. & Years of Service Nurse's & 0,165 & 0,280 & 1,323 & Not Significant \\
\hline 3. & Extra Income & 0,317 & 0,000 & 1,000 & Not Significant \\
\hline 4. & Head Family Position &. & 0 &. & Not Significant \\
\hline
\end{tabular}

c. Analysis Individual Characteristic Effect Of Nurse's Practic License (SIP) Nurse's

Table 5.81 Summary Multinomial Logistic Regression Statistical Test Results Individual Characteristic of Nurse's Practic License(SIP) Nurse's

\begin{tabular}{|l|l|r|r|r|l|}
\hline No & \multicolumn{1}{|c|}{ Variable' } & $\mathrm{p}$ & \multicolumn{1}{c|}{$\beta$} & \multicolumn{1}{c|}{$\mathrm{RR}$} & \multicolumn{1}{|c|}{ Information } \\
\hline 1. & Age's Nurse's & 0,466 & $-0,109$ & 0,897 & Not Significant \\
\hline 2. & Years of Service Nurse's & 0,165 & 0,280 & 1,323 & Not Significant \\
\hline 3. & Extra Income & 0,317 & 0,000 & 1,000 & Not Significant \\
\hline 4. & Head Family Position &. & 0 &. & Not Significant \\
\hline
\end{tabular}

N. Analysis Individual Characteristic Effect and Competition of Nurse's Of Service Quality Nurse's In the Inpatient Instalation

Table 5.82 Summary Multinomial Logistic Regression Statistical Test Results Individual Characteristic Nurse's Of Service Quality Nurse's

\begin{tabular}{|l|l|r|r|r|l|}
\hline No & \multicolumn{1}{|c|}{ Variable's } & \multicolumn{1}{c|}{ P } & \multicolumn{1}{c|}{$\beta$} & \multicolumn{1}{c|}{ RR } & Information \\
\hline 1. & Age's Nurse's & 0,089 & 0,318 & 1,374 & Not Significant \\
\hline 2. & Years of Service & 0,055 & $-0,393$ & 0,675 & Not Significant \\
\hline 3. & Extra Income & 0,115 & 0,000 & 1,000 & Not Significant \\
\hline 4. & Position & 0,755 & -0.474 & 0,623 & Not Significant \\
& Kepala & & & & \\
\hline 5. & Education of & 0,246 & $-0,833$ & 0,435 & Not Significant \\
\hline 6. & Trining & 0,699 & 0,032 & 1,003 & Not Significant \\
\hline 7. & Hard Skill & 0,132 & $-1,771$ & 0,170 & Not Significant \\
\hline 8. & Soft Skill & 0,005 & 0,024 & 1,025 & Significant \\
\hline 9. & Work Ethics & 0,423 & $-0,014$ & 0,986 & Not Significant \\
\hline 10. & Competence Result &. & $-20,836$ & $8,935 \mathrm{E}-10$ & Not Significant \\
\hline 11. & STR & 0,204 & $-1,213$ & 0,297 & Not Significant \\
\hline 12. & SIP &. & 0 &. & Not Significant \\
\hline
\end{tabular}

\section{DISCUSSION}

1. Analysis of Individual Characteristics and Their Influence on the Quality of Nurse Services in the Inpatient Room of Buleleng Hospital

Individual characteristic factors examined in this study are the age of the nurse, the length of service of the nurse, extra income and the position of head of the nurse's family. The age of nurses 
who worked in the inpatient room of Buleleng Hospital was at the most age range between 26 to 30 years, which was $61.3 \%$. At that age range, usually starting a career, starting a family life and starting caring for children. Changes in lifestyle from one's own family to more or less affect the quality of services provided while working. In Indonesian culture, women are identified with domestic work. Nurses in the inpatient ward of Buleleng Hospital, all of which are women, will certainly experience a change in role in the family, at the same time the obligation as a nurse working in a hospital is also demanded to be able to perform well.

The working period of nurses in the inpatient room of Buleleng Regional Hospital is mostly in the range of less than 5 years, which is $63.7 \%$. In the relatively new working period, the time obtained by new nurses is considered very sufficient time to adjust to the existing work environment. For nurses who first entered work in Buleleng, the orientation period was given three months. After evaluating, it will be decided whether to enter the contract stage or not. The first contract is valid for 1 (one) year. After completion of the first contract period there will be a reevaluation. The nurse in question will be decided to proceed at the second stage of the contract for 1 (one) year or not. After completing the second contract period, it will be decided to be appointed as a permanent employee or dismissed.

All nurses in the Inpatient Installation are female. This is intended so that the nature of caring and tenderness in the female side is more prominent in the care of patients in the Inpatient Installation. Cultural background in Indonesia, that women do not have the full obligation in making a living. The husband or head of the family plays the main role in finding sustenance for the family. Strong financial capacity in the hands of husband or father to unmarried nurses can provide different intrinsic motivation (lower) to nurses in carrying out their nursing duties.

The background of the position of head of the family, as well as extra income, is assumed to change the intrinsic motivation of a nurse at the executive level. The higher the position of head of the family, it is assumed that pride (pride) and a higher level of prestige compared to other nurses. With this background, it is assumed that there is an influence between the head of the family's debate on the quality of services provided. Of the total nurses studied, the status of the position of head of the family at most was at the executive level of $61.3 \%$.

From the multinomial logistic regression test of individual characteristics to nurse competence, the position of head of the family variable has a significant influence on aspects of work ethics. The significance figure obtained was 0,000 with RR 1794880,59. From these results it can be concluded that the higher the position of the head of a nurse's family, the work ethics he has will also be higher. This can be due to the pride of the nurse in question for the position of head of the family. From the results of multinomial regression tests conducted between individual characteristics of service quality, the significance figure shows the results of 0.586 . This means that there is no relationship between the position of the head of the nurse's family's work to the quality of services provided inpatient installations. As with extra income, this is possible because the responsibility for services provided to nurses has been absorbed by individual nurses with different levels 
2. Analysis of Nurse Competence and Its Effect on Nurse Service Quality in Inpatient Installations

The nurse competency factors examined in this study are basic knowledge, hard skills, soft skills, work ethics and legal aspects of each. Basic knowledge variables as part of nurse competence consist of 2 sub-variables, namely education that has been taken by the nurse concerned and training that has been undertaken by nurses. The ability of a nurse in completing her daily technical nursing duties is absolutely mastered by the nurse in question. There are 3 (three) types of nursing care in Buleleng inpatient rooms, namely surgical medical care, child care and obstetrics. Every nursing care requires mastery of technical abilities that differ from one another. Buleleng has established a minimum standard of technical mastery ability based on the competency standards of PPNI.

Soft Skill. The mastery of non technical skills is needed by nurses in completing daily work. Non-technical abilities include personal effectiveness, flexibility, leadership, persuasion, goal orientation, decision making, negotiation, analytical problem solving, teamwork, diplomacy, empathy, interpersonal skills and self management (time and priorities). The role of soft skills is important to master in today's work world (Heckman \& Kautz, 2; Andrews \& Higson, 2008). Employees who have better soft skills tend to be more successful in their jobs. Work Ethics. The work ethic of a nurse is closely related to the quality of services provided (Segall, 2000). The better the work ethic, the better the quality of service provided. Work ethics components in this study include self reliance, morality, leisure, hardwork, centrality of work, wasted time and delay of gratification.

The legal aspect of the competency is based primarily on Law number 36 year 4 concerning Health Workers and Law number 38 year 4 on Nursing. In carrying out professional practice nurses are required to be able to meet the legal aspects of a Practice License (SIP). While one of the requirements to get a SIP is to have a Registration Certificate (STR).

\section{Quality Analysis of Inpatient Installation Nurse Services}

Based on the results of the study, the quality of nurses' services in the Inpatient installation was assessed by patients in the poor category, which amounted to $47.5 \%$. Knowledgeability attitude in this study is shown by the attitude and behavior of nurses who are able to answer questions that are relevant to nursing care. Responsive attitude in this study is shown by the attitude and behavior of nurses who are responsive to the needs of patients. The attitude of professionalism referred to in this study is the attitude and behavior shown by maintaining skills, appearance, listening and manners. The attitude of accessibility in this study is shown by the attitudes and behaviors that provide convenience or contact or the meeting of officers. Caring in this study is shown by the attitudes and behaviors of nurses who listen to complaints, and are sensitive to the needs of patients.

The attitude of dignity in this study is shown by the attitudes and behavior of nurses who respect Seeta respecting the dignity of patients in each of their interactions. Emphaty attitude in this study is shown by the attitude and behavior of nurses who are able to position themselves in the patient's condition. The attitude of convenience in this study is shown by the attitudes and behaviors that provide comfort to the patient. The informative attitude referred to in this study is the attitude and behavior of nurses who always provide information without being asked and provide answers in accordance with their nursing duties. Communication attitude in this study is indicated by nurses' attitudes and behavior that convey information both verbally and non verbally clearly about the patient's needs.

\section{CONCLUSION}

1. In the soft skill variable, most nurses inpatients in Buleleng Hospital are still categorized as having poor soft skills. All soft skill subvariables, namely, personal effectiveness, flexibility, leadership, persuasion, goal orientation, decision making, negotiation, analytical problem solving, teamwork, diplomacy, empathy, interpersonal skills, and self management (time and priorities) are all poorly categorized. In this soft skill variable statistically influences the quality of nurse services.

2. In the work ethics variable, most of the nurses inpatient in Buleleng General Hospital have bad work ethics. All components of work ethics, namely self reliance, morality, leisure, hardwork, centrality of work, wasted time, and delay of gratification are categorized as poor. Statistically the work ethic of nurses in the inpatient room of Buleleng Hospital does not have an influence on the quality of services provided. 
3. In the legal aspect, most nurses are able to pass the competency test with just one exam. For the Registration Certificate (STR), some nurses have expired. Likewise, the Practice License (SIP), because the validity period is in accordance with the validity period of STR, some nurses have also expired. Statistically, this legal aspect is not related to the quality of nurse services in the inpatient room of Buleleng General Hospital.

4. The recommendations of this study are:

a. Making a good employee orientation system before work.

b. Preparation of technical guidance to new nurses who focus on daily experience.

c. Re-formulation of training objectives.

d. Development of guidelines for developing soft skills at the work unit level.

e. Establishment of a guidance and supervision system primarily related to direct services to patients.

f. Making a standard career path.

g. Building a work ethics culture with good standards

\section{DAFTAR PUSTAKA}

Andrews, J. \& Higson, H., 2008. Graduate Employability, 'Soft Skills' Versus 'Hard' Business Knowledge: A European Study. Higher Education in Europe, 33(4), pp.411-22.

Azwar V., 3. Peranan Moderasi Soft Skills dalam Meningkatkan Mutu Pelayanan Rumah Sakit. Jurnal Kesehatan Masyarakat Nasional, 7(8), pp.378-84.

Behrman, R., Kliegman, R. \& Nelson, W., 2002. Nelson Textbook of Pediatrics. 19th ed. Philadelphia: WB Saunders.

Blood, M.R., 1969. Work Values and Job Satisfaction. Journal of Applied psychology, 53(6), pp.456-59.

Cameron, K.S. \& Quinn, R.E., 2006. Diagnosing and Changing Organizational Culture. San Francisco: Jossey-Bass.

Cohen, J. \& Ezer, T., 3. Human Rights In Patient Care: A Theoretical And Practical Framework. Health And Human Rights, 15(2), pp.7-19.

Depkes, 0. Keputusan Menteri Kesehatan Nomor 1239 Years 2001 tentang Registrasi dan Praktik Nurse's. Jakarta: Departemen Kesehatan RI

Desmond, C. et al., 2002. Approaches to Caring, Essential Elements for A Quality Service and Cost-Effectiveness in South Africa. Evaluation and Program Planning, 25, pp.447-58.

Ginting, R., 1999. Peranan Pemimpin Informal Dalam Menggerakkan Partisipasi Masyarakat Untuk Pembangunan Desa. In Disertasi. Program PascaBachelor, Institut Pertanian Bogor.

Hatfield, N.T., 2008. Broadribb's Introductory Pediatric Nursing. 7th ed. Philadelphia: Lippincott Williams \& Wilkins.

Jacobalis, S., 1995. Liberalisasi Bisnis Jasa Kesehatan dan Dampaknya Bagi Rumah Sakit Indonesia. Jakarta: IRSYAM XXXVII.

Harper Collins. Knowles, M.S., Holton, E.F. \& Swanson, R.A., 5. The Adult Learner: The Definitive Classic in Adult Education and Human Resource Development. New York: Routledge.

Kotler, P. \& Keller, K.L., 2. Marketing Management. 14th ed. New Jersey: Prentice Hall.

Lewis, B.R. \& Mitchell, V.W., 2007. Defining and Measuring the Quality of Customer Service. Marketing Intelligence \& Planning, 8(6), pp.11-17.

Liaschenko, J. \& Peter, E., 2004. Nursing Ethics and Conceptualizations of Nursing: Profession, Practice and Work. Journal of Advanced Nursing, 46(5), pp.488-95.

Masdar, S., 2009. Manajemen Sumber Daya Manusia Berbasis Kompetensi. Surabaya: Airlangga Univeristy Press.

Miller, M.J., Woehr, D.J. \& HUdspeth, N., 2002. The Meaning and Measurement of Work Ethic: Construction and Initial Validation of a Multidimensional Inventory. Journal of Vocational Behavior, 60, pp.451-89.

Nelson, J.A. \& Folbre, N., 2006. Why a Well-Paid Nurse is a Better Nurse. Nurs Econ, 24(3), pp.127-30.

Omachonu, V.K. \& Ross, J.E., 2005. Principles of Total Quality. 3rd ed. New York: CRC Press. 
Petersen, L.A. et al., 2006. Does Pay-for-Performance Improve the Quality of Health Care? Annals of Internal Medicine, 145(4), pp.265-72.

PPNI, 0. Standar Profesi dan Kode Etik Nurse's. Jakarta: PP PPNI

Robbins, S.P. \& Judge, T.A., 3. Organizational Behavior. 15th ed. New York: Pearson.

Rosander, A.C., 1985. Applications of Quality Control in the Service Industries. New York: CRC Press.

St-Germain, D., Boivin, B. \& Fougeyrollas, P., 1. The Caring-Disability Creation Process Model: a New Way of Combining 'Care' in Nursing and 'Rehabilitation' for Better Quality of Services and Patient Safety. Disability and Rehabilitation, 33(21-22), pp.2105-13.

Timby, B.K. \& Smith, N.E., 0. Introductory Medical-Surgical Nursing. 10th ed. Philadelphia: Lippincott Williams \& Wilkins.

Wijono, D., 2008. Manajemen Mutu Rumah Sakit dan Kepuasan Pasien. Surabaya: Airlangga University Press.

Yu, G., Wang, Y. \& Liu, C., 2. Improving Public Service Quality from a Developmental Perspective: Empathy, Attachment, and Gender Differences. Pubic Personnel Management, 41(5), pp.9-20.

Zelenski, J.M., Murphy, S.A. \& Jenkins, D.A., 2008. The Happy-Productive Worker Thesis Revisited. J Happiness Stud, 9, pp.521-37. 